\title{
Bounds of percolation thresholds in the enhanced binary tree
}

\author{
Seung Ki Baek*, Petter Minnhagen \\ Integrated Science Laboratory, Department of Physics, Umeå University, 90187 Umeå, \\ Sweden
}

\begin{abstract}
By studying its subgraphs, it is argued that the lower critical percolation threshold of the enhanced binary tree (EBT) is bounded as $p_{c 1}<0.355059$, while the upper threshold is bounded both from above and below by $1 / 2$ according to renormalization-group arguments. We also review a correlation analysis in an earlier work, which claimed a significantly higher estimate of $p_{c 2}$ than $1 / 2$, to show that this analysis in fact gives a consistent result with this bound. Our result confirms that the duality relation between critical thresholds does not hold exactly for the EBT and its dual, possibly due to the lack of transitivity.
\end{abstract}

Keywords: percolation threshold, enhanced binary tree, hyperbolic lattice 2000 MSC: 82B43, 51P05

\section{Introduction}

Percolation has been always of physical interest since it was introduced as a description for a fluid in random media [1]. The percolation problem has served as one of the basic models in understanding critical phenomena from a geometric viewpoint [2]. An interesting aspect of the percolation phenomenon is that a lattice with a constant negative curvature may have two different thresholds, which coalesce into one as the curvature vanishes [3] . Figure 1(a) gives the simplest example of a hyperbolic lattice: a regular tree with level $L$, where $L$ is defined as the maximum path length from the

\footnotetext{
${ }^{*}$ Corresponding author

Email address: garuda@tp.umu.se (Seung Ki Baek)
} 
origin. The set of points at the same distance $l$ from the origin constitutes the $l$ th layer. In this tree, every vertex has three neighbors except at the boundary, and the number of vertices scales as $N \sim 2^{L}$. An immediate consequence is that the number of boundary points, which scales as $B \sim 2^{L-1}$, always occupies a finite fraction of $N$ even in the thermodynamic limit. The existence of two percolation thresholds has been attributed to this peculiar structural property. That is, when the occupation probability $p$ reaches the lower threshold, $p_{c 1}$, a system-wide connection is first achieved, while the largest cluster occupies a finite fraction of $N$ only if $p$ exceeds the upper threshold, $p_{c 2}$. Therefore, if we count the boundary points connected to the midpoint of the system as we increase $p$, the connection simply does not exist at $p<p_{c 1}$, and even after passing this first threshold the connected boundary points remain as a negligible part of the whole boundary until $p$ reaches $p_{c 2}$. In the case of the bond-percolation problem in a simple tree as in Fig. 11(a), for example, one can easily show that $p_{c 1}^{\text {tree }}=1 / 2$ and $p_{c 2}^{\text {tree }}=1[3]$.

The enhanced binary tree (EBT) is a nontrivial model derived from a tree [4]: it is obtained by adding bonds to the tree between every pair of neighboring points within each layer $l>1$ [Fig. 1)(b)], so it has $p_{c 1}<p_{c 1}^{\text {tree }}$ and $p_{c 2}<p_{c 2}^{\text {tree }}$ due to the existence of loops. An interesting analogy of an EBT would be a biological taxonomy which is mostly a tree structure but with genes horizontally transferred as well (see, e.g., [5]). Although the lower threshold could be easily measured as $p_{c 1}=0.304(1)$ [4], there remains a controversy in locating $p_{c 2}$ [4, 6, 7]: [4] claimed that $p_{c 2}=0.564(1)$, and the correlation analysis was suggested to support this claim [7], while other numerical methods preferred $p_{c 2}=0.48(1)$ [6]. Recently, there appeared an analytic calculation [8], which suggests a possible generalization of the triangle-triangle transformation [9, 10, 11]: in order to use this transformation in an exact fashion, one should be able to decompose a given structure of identical triangular unit cells, where the three vertices of one unit cell are denoted as $A, B$, and $C$, respectively (see Fig. 2(a)). Then $P(A, B, C)$ is defined as the probability that $A, B$, and $C$ are all connected, $P(\bar{A}, B, C)$ as the probability that $B$ and $C$ are connected but $A$ is disconnected from them, and $P(\bar{A}, \bar{B}, \bar{C})$ as the probability that none of them are connected to each other. One may locate the critical threshold by equating these two probabilities [10]:

$$
P(A, B, C)=P(\bar{A}, \bar{B}, \bar{C}) .
$$

From a trivial equality, $P(\bar{A}, \bar{B}, \bar{C})+P(A, B, \bar{C})+P(A, \bar{B}, C)+P(\bar{A}, B, C)+$ 


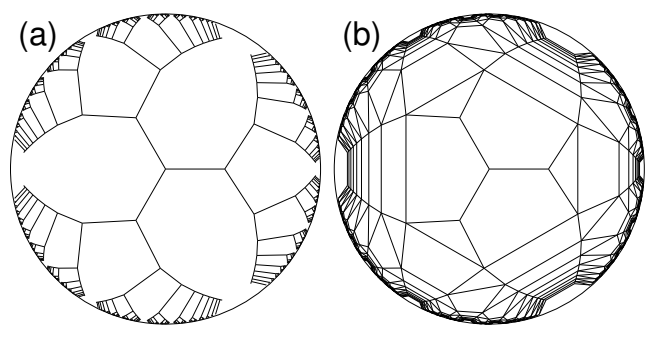

Figure 1: Schematic plots of hyperbolic structures drawn on the Poincaré disk. (a) A tree structure up to level $L=10$ and (b) the enhanced tree obtained from (a). The midpoint is located at the origin in each plot.

(a)

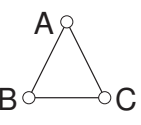

(b)

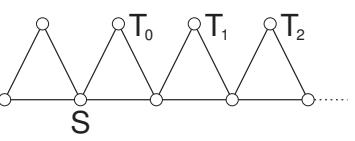

Figure 2: (a) A triangular cell having vertices $A, B$, and $C$. (b) An array of such triangular cells, where the $T_{i}$ indicate the points in the upper layer on the right-hand side of $S$.

$P(A, B, C)=1$, one can express Eq. (1) as

$$
\frac{P(A, B)}{1-P(\bar{A}, B, C)}+\frac{P(A, C)}{1-P(\bar{A}, B, C)} \equiv \Psi=1,
$$

where $P(\alpha, \beta)$ means the probability of connection between vertices $\alpha$ and $\beta$. If one picks up an arbitrary starting point $S$ in a very large layer as in Fig. 2(b), the probability of connecting to the upper layer on its right-hand side can be written as $\sum_{i=0}^{\infty} \operatorname{Prob}\left(T_{i}\right.$ is the first right-hand connection $)=$ $\sum_{i=0}^{\infty} P(A, B) P^{i}(\bar{A}, B, C)$, which is identical to the first term in Eq. (2). Likewise, the second term in Eq. (2) means the probability of a left-hand connection from $S$. In this sense, Eq. (1) can be regarded as describing a certain connective property between two adjacent layers at criticality (see also [12], where one finds a similar idea). Applying this idea to the EBT without requiring the self-duality of the triangular type, one obtains $\Psi(p)=$ $p(1+p) /[1-p(1-p)][8]$. Then, the equation $\Psi(p)=1$ is satisfied at $p=1 / 2$, which in [8] was interpreted as an exact value of $p_{c 2}$ for the EBT. Since the simple binary tree has $\Psi^{\text {tree }}\left(p_{c 1}^{\text {tree }}\right)=1 / 2$, assuming this again holds for the EBT, one obtains the value of $p_{c 1}$ as $(\sqrt{13}-3) / 2 \approx 0.302776$ [8]. 
(a)

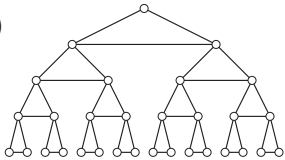

(b)

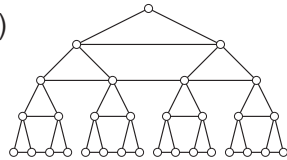

(c)

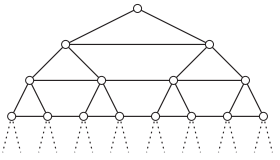

Figure 3: Subgraphs of the EBT for bounding $p_{c 1}$. (a) The tree made of triangles has its lower threshold at $p_{1}^{*} \approx 0.403032$, which bounds $p_{c 1}$ from above. (b) By adding more bonds at every second layer, a larger unit cell is constructed, and we get a sharper bound, $p_{2}^{*} \approx 0.373897$. (c) The largest unit cell considered in this work, giving the upper bound $p_{c 1}<p_{3}^{*} \approx 0.355059$. The dotted lines mean that other unit cells are attached there.

In this work, we use alternative approaches and argue that $p_{c 1}<0.355059$ by examining solvable subgraphs and $p_{c 2} \leq 1 / 2$ by means of the renormalizationgroup (RG) method on hierarchical structures [13]. We also demonstrate that the correlation analysis indeed gives a consistent result with this argument for $p_{c 2}$. The paper is organized as follows. We explain the results for $p_{c 1}$ and $p_{c 2}$ in Sec. 2 and Sec. 3, respectively. Then Sec. 4 additionally discusses the correlation behavior, and Sec. [5 summarizes this work.

\section{Lower threshold}

A critical percolation threshold becomes higher when part of the links are removed from the original graph. We could therefore argue that $p_{c 1}<1 / 2$ above by considering the simple binary tree as a subgraph of the EBT. We refine this bound by taking larger but still solvable subgraphs.

One may first consider a tree of triangles, which is also a subgraph of the EBT (see Fig. 3(a)). We are going to ask whether there can be any path penetrating from the top to the bottom. A relevant quantity would then be how many vertices can be reached on the $(l+1)$ th layer from a single vertex on the $l$ th layer. If we focus on a unit cell, which is simply a triangle here, we find $2^{3}$ possible configurations, since it has three bonds. By checking all of these configurations, one can easily get the average number of descendants, i.e., the expected number of bottom points connected to the top vertex within the cell. It is easily given as

$$
n_{1}(p)=2 p(1-p)^{2}+6 p^{2}(1-p)+2 p^{3}=2 p[1+p(1-p)] .
$$

Solving $n_{1}\left(p_{1}^{*}\right)=1$, we obtain $p_{1}^{*}=\frac{1}{3}(1+2 \cos \theta-2 \sqrt{3} \sin \theta) \approx 0.403032$, 
where $\theta \equiv \frac{1}{3} \arctan \left(\frac{9 \sqrt{37}}{5 \sqrt{3}}\right)$. This provides an improved upper bound for $p_{c 1}$. Note also that $n_{1}(p)$ is a monotonically increasing function of $p$ from zero to 2. This implies that the upper threshold of this subgraph is located at $p=1$, since otherwise the fraction of connected vertices will decrease as the number of passing layers increases.

This sort of construction requires that a unit cell should possess only one top vertex and that every bottom-layer point equally becomes a new top vertex for a subsequent unit cell. We can therefore extend this idea a little further, as follows. For every second layer, we add a bond between two daughter triangles under the same mother triangle, as in Fig. 3(b). This creates a new unit cell containing three triangles plus one bond between the daughter cells. Such a cell has 10 bonds in total, meaning $2^{10}=1024$ possible configurations. Directly enumerating them shows again how the top vertex connects to the bottom layer within this cell. The average number of descendants then reads

$$
n_{2}(p)=2 p^{2}[1+p(1-p)]\left[2+p(1-p)\left(3+3 p+p^{2}-10 p^{3}+5 p^{4}\right)\right],
$$

which ranges over $\left[0,2^{2}\right]$. Numerically solving $n_{2}\left(p_{2}^{*}\right)=1$ leads to $p_{2}^{*} \approx$ 0.373897. It is still possible to consider a larger unit cell with seven triangles (see Fig. 3(c)) and find the average number of connected bottom-layer points as

$$
\begin{aligned}
n_{3}(p)= & 2 p^{3}[1+p(1-p)]\left[4+p(1-p)\left(13+26 p+23 p^{2}-37 p^{3}-64 p^{4}\right.\right. \\
& -42 p^{5}-116 p^{6}+234 p^{7}+469 p^{8}-830 p^{9}+1811 p^{10}-2898 p^{11} \\
& -4735 p^{12}+21801 p^{13}-31538 p^{14}+24399 p^{15}-10894 p^{16} \\
& \left.\left.+2664 p^{17}-278 p^{18}\right)\right]
\end{aligned}
$$

from $2^{25}$ configurations. By solving $n_{3}\left(p_{3}^{*}\right)=1$, we find an upper bound for $p_{c 1}$ as $p_{3}^{*} \approx 0.355059$. On the other hand, it is readily seen that $p_{c 1}$ can never be lower than 0.25 , since every vertex in the EBT has $z=5$ neighbors except the zeroth layer, so that the tree approximation yields $1 /(z-1)=1 / 4$. We note that both the numerically obtained threshold $p_{c 1}=0.304(1)$ [4] and the analytic prediction $p_{c 1}=(\sqrt{13}-3) / 2 \approx 0.302776$ [8] lie within these upper and lower bounds. Since this subgraph method soon becomes impractical due to the huge number of possible configurations as the size of a unit cell grows, there is no sharper bound available at present. One may try an extrapolation by using the correlation-length scaling exponent $\nu=1$ at $p=p_{c 1}$ [3], but this 
(a)

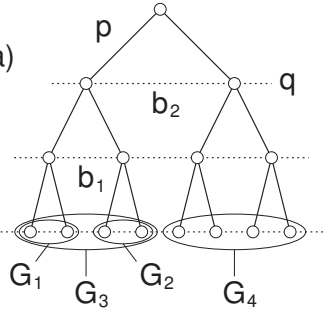

(b)

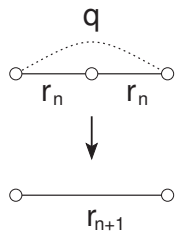

(d)

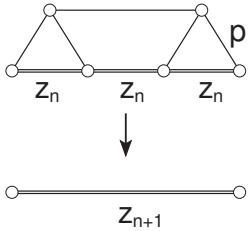

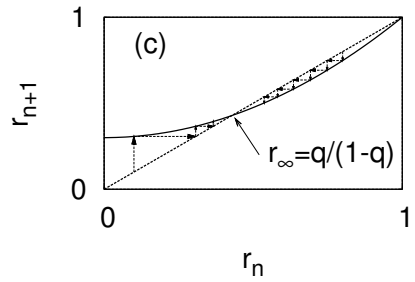

$r_{n}$

Figure 4: (a) A part of an EBT structure. The tree part (solid) is occupied with probability $p$ and the rest of the bonds, i.e., the horizontal lines, are occupied with probability $q$. At $p=1 / 2$, a single bond $b_{1}$ will directly connect two groups of boundary points, $G_{1}$ and $G_{2}$, with probability $q$, while $b_{2}$ will connect $G_{3}$ and $G_{4}$. (b) Schematic description for $r_{n+1}$, the global-connection probability for the bottom layer at the $(n+1)$ th RG iteration. Hence the probability $r_{n}$ at the $n$th iteration is related to $r_{n+1}$ by Eq. (3). (c) Dynamics of the RG flow for $q \leq 1 / 2$ according to Eq. (3), which is here drawn as the solid line. (d) An alternative recursion scheme illustrating Eq. (4), where the double lines represent coarse-grained links, i.e., $z_{n}$ or $z_{n+1}$.

will be a rather crude estimation. It is also clear that these subgraphs do not delimit $p_{c 2}$ of the EBT at all since all of them have their upper thresholds at $p=1$.

\section{Upper threshold}

The main idea in bounding $p_{c 2}$ begins with the fact that a simple binary tree has a lower threshold $p_{c 1}^{\text {tree }}=1 / 2$. We assign occupation probabilities $p$ and $q$ to the tree part and the rest of the EBT, i.e., the horizontal bonds in Fig. 四(a), respectively. Let us start from $q=0$, where the EBT becomes identical to a simple tree. At $p=1 / 2$, the tree part reaches its first critical threshold so that each vertex is connected to one boundary point on average. Then we increase $q$ so that a finite fraction of boundary points can merge into a single cluster. 
An interesting property of the EBT is that the horizontal bonds would have different connection ranges depending on which layer they belong to. In Fig. 4(a), bond $b_{1}$ directly connects two groups of boundary points, $G_{1}$ and $G_{2}$, at average distance 2, which is measured along the bottom layer. On the other hand, the average range of connection between groups $G_{3}$ and $G_{4}$ by bond $b_{2}$ is twice as long as than that. In other words, if we focus only on the bottom layer by setting $p=1 / 2$, the horizontal bonds constitute a hierarchical structure so that the range of connections becomes twice as long every time when a new higher level is introduced. Such a property allows one to formulate an RG equation [13], which here can be written as

$$
r_{n+1}=q+(1-q) r_{n}^{2},
$$

where $r_{n}$ is the probability of global connection in the bottom layer at the $n$th RG scaling transformation (see Fig. 4(b)). Here, we are asking ourselves how probable it is for two different points that we have arbitrarily picked up from the boundary bottom layer to belong to the same cluster. Since a cluster containing the middle part of the system occupies only a negligible fraction of the whole boundary at $p<p_{c 2}$ by definition, this means that such a chance gets significantly large above $p_{c 2}$, and thereby Eq. (3) is related to the upper threshold. It is stated in Eq. (3) that the global connection can be established either by a new long link with probability $q$ or by existing shorter links. In the limit of large $n$, we may set $r_{n}=r_{n+1}=r_{\infty}$ and then Eq. (3) is easily solved to yield a nontrivial stable solution, $r_{\infty}=q /(1-q)$, as shown in Fig. 4(c) [13]. Noting that $r_{\infty}$ is responsible for connecting a number of the boundary points to one another at criticality, we find that $q=1 / 2$ should be a transition point provided that $p$ is fixed at $p_{c 1}^{\text {tree }}=1 / 2$. Since $p$ and $q$ happen to have the same value here, even if considering the homogeneous case where $q$ is always set equal to $p$, we can conclude that $p=1 / 2$ is high enough to connect a significant fraction of boundary points. In short, $p=1 / 2$ should be higher than or equal to $p_{c 2}$, the upper critical percolation threshold of the EBT.

We note that Eq. (3) is an approximate description with a coarse-grained variable $r_{n}$, chosen for ease of explanation. It is possible to make an alternative recursion scheme which instead yields a lower bound. The scheme is illustrated in Fig. 4(d), where $q$ is assumed to be equal to $p$. There are two outer points on the left-hand side and two others on the right-hand side. In merging a set of bonds into a single one with connection probability $z_{n+1}$, we are interested in linking any of the left outer points to any of the right outer 
points. Obviously, a part of contribution to $z_{n+1}$ comes from filling the upper bond with probability $p$. Even if it is not filled with $1-p$, there remain a few more possible cases. Suppose that there happen to be two filled bonds which are the right one of the left triangle and the left one of the right triangle, for example. It is then enough to have only one $z_{n}$ in between. Likewise, we can consider all the other cases and arrive at the following recursion relation:

$$
z_{n+1}=p+(1-p)\left[(1-p)^{2} z_{n}^{3}+2 p(1-p) z_{n}^{2}+p^{2} z_{n}\right] .
$$

Solving this by setting $z_{n}=z_{n+1}=z_{\infty}$ as above, one finds that

$$
z_{\infty}=\frac{\sqrt{1+4 p-6 p^{2}+p^{4}}}{2(1-p)^{2}}-\frac{1+p}{2(1-p)} .
$$

Again, we see that $z_{\infty}=1$ at $p \geq 1 / 2$. However, since the renormalization includes connections between the lower outer points even if there is no interlayer connection, the result yields a lower bound of the threshold.

\section{Correlation Analysis}

A recent numerical estimation in [7] suggested $p_{c 2}>0.56$, which certainly exceeds $1 / 2$ given above as an upper bound. We present a brief discussion on this discrepancy. Following [7], let us consider the probability $C(l)$ that points at level $l$ in the EBT belong to the same cluster as the midpoint does. Such a correlation will be a monotonically decreasing function of $l$, and it converges to a constant if $p>p_{c 2}$. If we are to take only robust behavior insensitive to any particular $L$, a possible way would be to compare two different system sizes, say $L=23$ and 24 , and take only data points up to $l=l_{\max }$, where they cease to overlap within error bars. Furthermore, we need to exclude $l<l_{\min }$, since the EBT has a lower number of connections close to the midpoint, so $C(l)$ may decrease anomalously at small $l$. For the data presented in Fig. 5, we set $l_{\min }=2$ for $p<0.55$ and $l_{\min }=1$ for $p \geq 0.55$ because of the more rapid exponential decay, which possibly implies that $\xi \lesssim 2$. We assume that $C(l)$ within the range of $l_{\min } \leq l<l_{\max }$ will show a simple decaying behavior with a certain characteristic length scale $\xi$ as

$$
C(l) \sim a \exp (-l / \xi)+c,
$$

where $a$ and $c$ are independent of $l$. The parameter $c$ can be also said to be the asymptotic value of correlation at large $l$. Since this term in the 

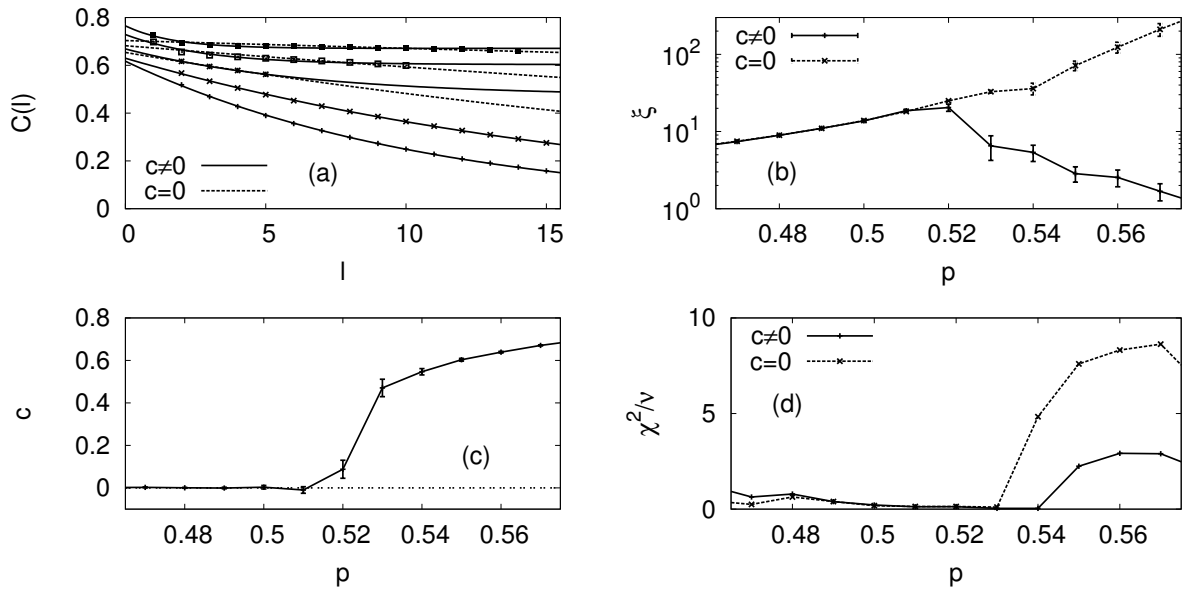

Figure 5: (a) Correlation function measured for $L=24$ over $10^{4}$ samples. The occupation probabilities are $p=0.49,0.51,0.53,0.55$, and 0.57 , from bottom to top. The solid curves are according to Eq. (5D) with least-square-fitted parameter values, while the dotted curves are found when we fit the data without $c$. (b) Correlation length $\xi$ obtained from the measurements. One observes a peak around $p=0.51$ from the fitting by Eq. (5) (solid). If one fits the same data after setting $c=0, \xi$ can be overestimated in the supercritical phase (dotted). (c) Asymptotic correlations obtained by Eq. (5). (d) Reduced $\chi^{2}$ values for $c \neq 0$ and $c=0$, respectively. 
fitting procedure was not included in [7], $\xi$ is presumably overestimated in the supercritical phase, since the nonvanishing part of $C(l)$ would be interpreted as a very slow decrease (see Fig. 55(a)). Our fitting results plotted in Fig. 5 (b) and Fig. 5)(c) show signatures of the transition around $p=0.51$, which is significantly lower than $p=0.56$ claimed in [7], and is in fact fairly close to $1 / 2$. In order to compare the goodness of fits, we calculate the $\chi^{2}$ statistic defined as

$$
\chi^{2}=\sum_{i} \frac{\left(O_{i}-E_{i}\right)^{2}}{\sigma_{i}^{2}},
$$

where $O_{i}$ is the $i$ th data point observed with variance $\sigma_{i}^{2}$, and $E_{i}$ is the corresponding expected value from Eq. (5). The number of degrees of freedom, $\nu$, is here given as the number of data points minus the number of fitting parameters. The reduced $\chi^{2}$ statistic, $\chi^{2} / \nu$, allows one to compare the performance of different fitting functions, and a rule of thumb states that a good fit is achieved when $\chi^{2} / \nu \sim O(1)$. In Fig. 5 (d), we find that including the asymptotic correlation term, $c$, indeed describes the behavior of $C(l)$ better, since it makes the reduced $\chi^{2}$ statistic maintain its value around $O(1)$ throughout the checked range of $p$, while the pure exponential function without $c$ becomes a poor description for the same data at $p \gtrsim 0.54$. One therefore finds that the threshold value determined numerically is consistent with the analytic prediction given above, although the result is subject to a larger uncertainty than the case of $p_{c 1}$.

\section{Summary}

In summary, we set upper bounds for $p_{c 1}$ and $p_{c 2}$ of the EBT structure; that is, $p_{c 1}<p_{3}^{*}=0.355059$ and $p_{c 2} \leq 1 / 2$. In addition, we obtained $p_{c 2} \geq 1 / 2$ as well, which confirms the argument in [8] that $p_{c 2}=1 / 2$. Since the upper threshold has been particularly under debate, we tried to settle the issue to a large extent by showing that the RG method developed for hierarchical structures led to the bounds of $p_{c 2}$. We also demonstrated that the correlation analysis yielded a consistent result with this RG argument. Both of these analytical and numerical approaches disprove the duality conjecture that the EBT and its dual lattice are related in such a way that the upper threshold for the EBT, $p_{c 2}$, and the lower threshold for the dual lattice, $\bar{p}_{c 1}$, sum up to $p_{c 2}+\bar{p}_{c 1}=1$ [4], since the lower threshold for the dual lattice is numerically determined as $\bar{p}_{c 1}=0.436(1)$ to good precision [4]. The possible 
value of $p_{c 2}$ given in this work clearly shows that $\bar{p}_{c 1}+p_{c 2}<1$, as already reported for other hyperbolic lattices [3]. This observation confirms that such a duality relation requires transitivity [14], which does not hold for the EBT.

From a methodological viewpoint, our analysis in this work relies largely upon the regularity of the underlying structure. There also exist other regular hierarchical structures such as the Apollonian networks [15, 16] and flower

networks [17], where the percolation problem has been studied by means of recursion. The extension and usefulness of our subgraph analysis for these cases remain to be investigated.

\section{Acknowledgement}

We are grateful to Dr. Sebastian Bernhardsson for discussions. We acknowledge the support from the Swedish Research Council with Grant No. 621-2002-4135. This research was conducted using the resources of High Performance Computing Center North (HPC2N).

\section{References}

[1] S. R. Broadbent, J. M. Hammersley, Percolation processes: I. crystals and mazes, Proc. Cambridge Philos. Soc. 53 (1957) 629.

[2] D. Stauffer, A. Aharony, Introduction to Percolation Theory, Taylor \& Francis, London, 2 edition, 2003.

[3] S. K. Baek, P. Minnhagen, B. J. Kim, Percolation on hyperbolic lattices, Phys. Rev. E 79 (2009) 011124.

[4] T. Nogawa, T. Hasegawa, Monte Carlo simulation study of the twostage percolation transition in enhanced binary trees, J. Phys. A 42 (2009) 145001.

[5] C. Gilbert, S. Schaack, J. K. Pace II, P. J. Brindley, C. Feschotte, A role for hostparasite interactions in the horizontal transfer of transposons across phyla, Nature 464 (2010) 1347.

[6] S. K. Baek, P. Minnhagen, B. J. Kim, Comment on 'Monte Carlo simulation study of the two-stage percolation transition in enhanced binary trees', J. Phys. A 42 (2009) 478001. 
[7] T. Nogawa, T. Hasegawa, Reply to the comment on 'Monte Carlo simulation study of the two-stage percolation transition in enhanced binary trees', J. Phys. A 42 (2009) 478002.

[8] P. Minnhagen, S. K. Baek, Analytic results for the percolation transitions of the enhanced binary tree, Phys. Rev. E 82 (2010) 011113.

[9] C. R. Scullard, Exact site percolation thresholds using a site-to-bond transformation and the star-triangle transformation, Phys. Rev. E 73 (2006) 016107.

[10] R. M. Ziff, Generalized cell-dual-cell transformation and exact thresholds for percolation, Phys. Rev. E 73 (2006) 016134.

[11] R. M. Ziff, H. Gu, Universal condition for critical percolation thresholds of kagomé-like lattices, Phys. Rev. E 79 (2009) 020102(R).

[12] M. Vuorio, A method to estimate the critical probability in bond percolation problems, J. Chem. Phys. 60 (1974) 846.

[13] S. Boettcher, J. L. Cook, R. M. Ziff, Patch percolation on a hierarchical network with small-world bonds, Phys. Rev. E 80 (2009) 041115.

[14] I. Benjamini, O. Schramm, Percolation in the hyperbolic plane, J. Am. Math. Soc 14 (2000) 487-507.

[15] J. S. Andrade, Jr., H. J. Herrmann, R. F. S. Andrade, L. R. da Silva, Apollonian networks: Simultaneously scale-free, small world, Euclidean, space filling, and with matching graphs, Phys. Rev. Lett. 94 (2005) 018702.

[16] D. M. Auto, A. A. Moreira, H. J. Herrmann, J. S. Andrade, Jr., Finitesize effects for percolation on Apollonian networks, Phys. Rev. E 78 (2008) 066112.

[17] H. D. Rozenfeld, D. ben-Avraham, Percolation in hierarchical scale-free nets, Phys. Rev. E 75 (2007) 061102. 